\title{
Intelligent Shutter Speech Control System Based on DSP
}

\author{
Deng Yonghong, Ge Yanxiang ,Zhao Zimei, \\ College of electronic and information engineering, North China Institute of Science and Technology, 065201
}

\begin{abstract}
Based on TMS320F28035 DSP, this paper designed a smart shutters voice control system, which realized the functions of opening and closing shutters, intelligent switching of lighting mode and solar power supply through voice control. The traditional control mode is converted to voice control at the same time with automatic lighting and solar power supply function. In the convenience of people's lives at the same time more satisfied with today's people on the intelligent and environmental protection of the two concepts of the pursuit. The whole system is simple, low cost, safe and reliable.
\end{abstract}

Key words: blind ,DSP, voice control, solar power supply, automatic chasing light, intelligent home, environmental protection

\section{Ask questions}

With the rapid development of today's society and the continuous improvement of people's living needs, the traditional home and residential has become increasingly unable to meet people's demand for intelligence, environmental protection, convenience, fast and comfortable. At present, life mainly rely on manual or electric to open and close the blinds, when the outdoor light changes also need to adjust the location of the blinds so that indoor light to maintain comfort. The blinds controlled by this mode will inevitably bring inconvenience to the user, especially those with reduced mobility. Waste people's time at the same time also delayed people's work. Therefore, this paper combines the current market demand, combined with engineering and practical application, designed this set of voice control, automatic chasing light, solar power supply three functions in one and can well meet the needs of people living green blinds.

\section{System composition}

\section{1 target function}

(1) Through the voice to control the opening and closing shutters
(2) When the outdoor light changes, the blinds will automatically adjust the current chase light mode, rotate the corresponding angle to achieve the desired effect

(3) In the shading at the same time the largest use of resources, through the fan on the solar panels to the battery charge

\subsection{System frame structure}

Figure 1 shows that the DSP-based intelligent shutters voice control system consists of a battery charging module, a stepper motor drive module, a voice module, a photoresistor sampling module and a TMS320F28035 main control board.

(1) battery charging module: mainly for the entire system to provide power support. The voltage on the solar panel is converted to the required voltage to power the system.

(2) stepper motor drive module: the main system to provide dynamic support.

(3) voice module: the main responsibility for the entire system of information feedback.

(4) photoresistor sampling module: is mainly responsible for the collection of external light signals and information back to the main control board.

(5) DSP main control panel.

Each module with each other, coordination of work, constitute a complete intelligent shutters voice control system. 


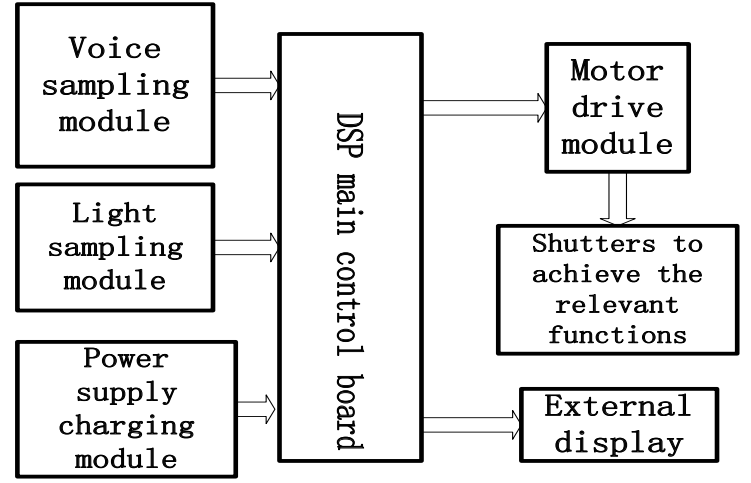

Figure 1 system overall frame structure

\section{Hardware circuit design}

\section{1 voice recognition module}

The voice module circuit is mainly based on LD3320 chip-free programming non-specific speech recognition module. The module will provide a specific return value to the main control board when a specific voice signal is

received. The main control board according to the different statements of different return value issued a different order so that the blinds to achieve different functions.

Speech recognition consists of two phases: training phase and recognition stage.

(1) training phase: through a number of voice input after preprocessing and feature extraction feature parameters obtained in order to establish a voice reference model library.

(2) identification stage: the input voice parameters and reference model library model similarity comparison, and the highest degree of similarity as a result of the output. LD3320 chip internal voice recognition principle: first of the input sound spectrum analysis, and then extract the voice feature to get the keyword information, and then the voice reference model of the data characteristics of the comparison, and ultimately find the best keywords and As a result of the output. Schematic diagram shown in Figure 2:

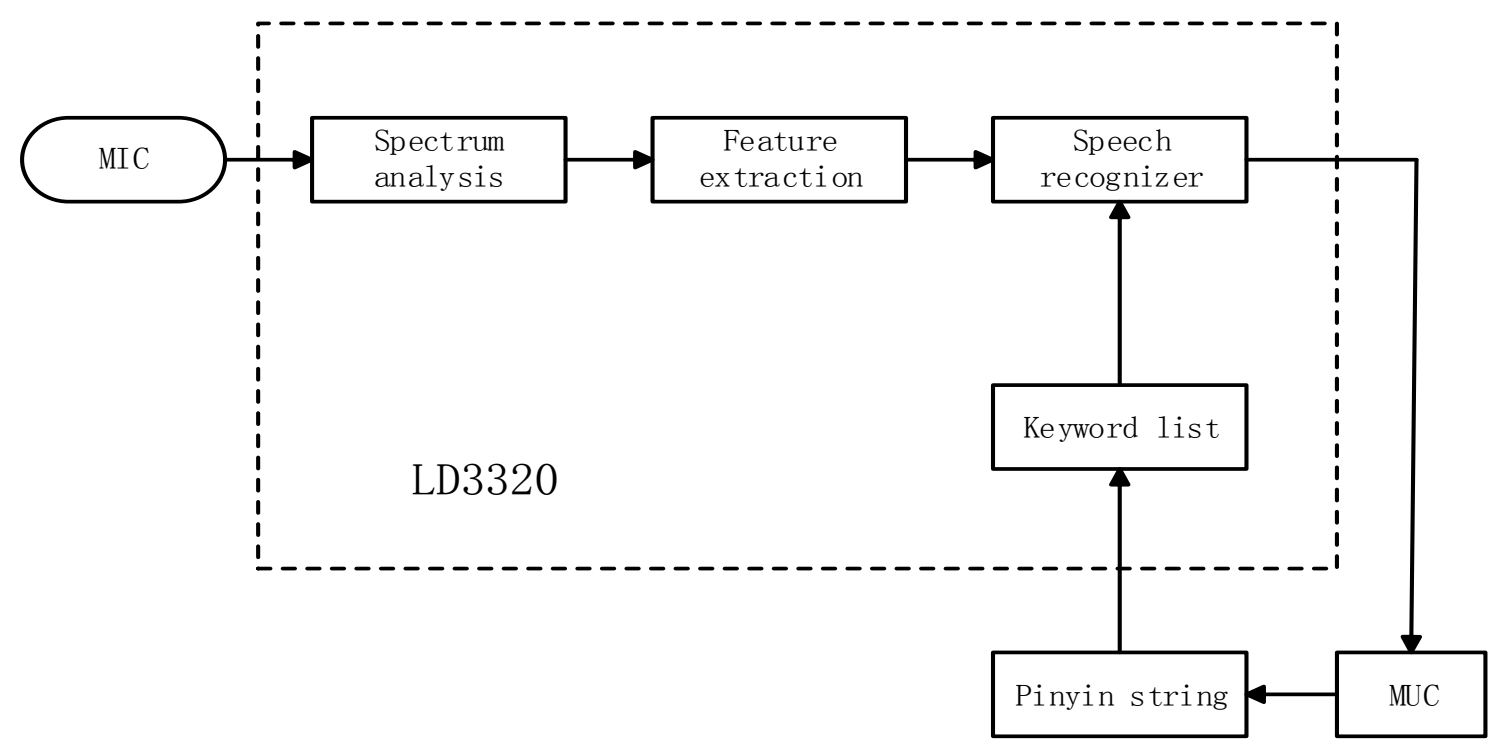

Figure 2 speech recognition module

\subsection{Motor drive}

The motor drive module is mainly for the entire system to provide power support, is to complete the motor action command executor. In order to ensure the use of shutters, we use the more precise step stepper motor as the drive motor, and ULN2003 chip composition shown below the motor drive circuit to drive, when the main control board to receive the signal will be implemented after the corresponding Action to ensure the realization of the various functions. 
P1

U1

U2

P2

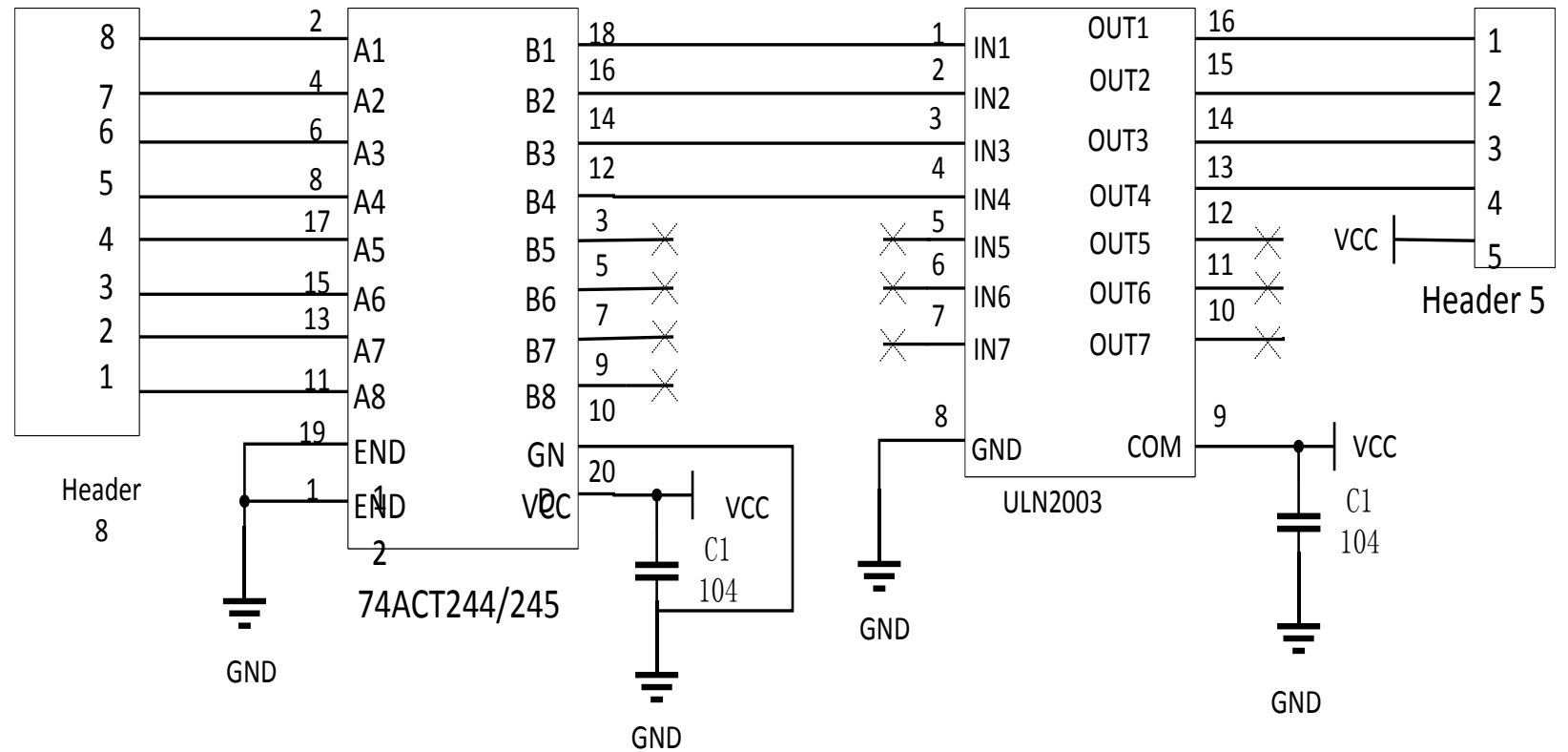

Figure 4 Schematic diagram of stepper motor drive

Mainly for the entire system to provide power support. To meet the needs we set the following two power supply.

(1) mains power supply: DC $5 \mathrm{~V}$ power supply or with a $5 \mathrm{~V}$ transformer to convert the mains of electricity to $5 \mathrm{~V}$ DC power supply for the entire system. Mainly used when the battery is fully charged or the solar power supply system can not be used normally.

(2) solar power: solar energy supply and environmental protection, the principle is mainly the use of fan blades on the solar panels to collect solar energy, and then use the CN3722 chip composed of solar cells with the maximum power tracking function and can effectively charge a number of lithium batteries Management of the circuit, to achieve the purpose of energy saving and environmental protection. The circuit diagram is as follows.

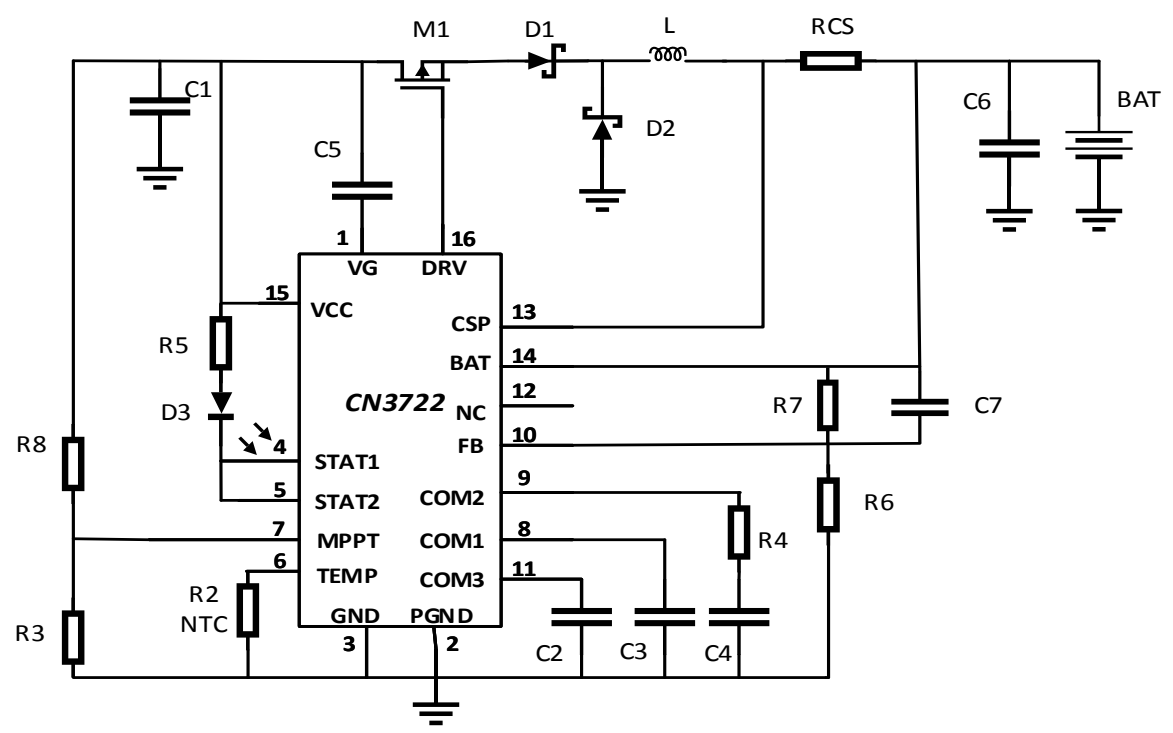

Figure $5 \mathrm{CN} 3722$ based on the battery charging circuit schematic

We use the CN3722 is a can use the solar power supply PWM decompression mode charge management integrated circuit, with solar cell maximum power point tracking function. CN3722 is very suitable for single or multi-cell lithium battery or lithium iron phosphate battery charge management, with a small package, small external components and the advantages of simple use. CN3722 with constant current and constant voltage charging mode, 
very suitable for lithium batteries or lithium iron phosphate battery charging. In constant voltage charging mode, the constant voltage charging voltage is set by the external resistor divider network; in the constant current charging mode, the charging current is set by an external resistor. For deep discharge batteries, the CN3722 charges the battery with $15 \%$ of the constant current charge current set when the battery voltage is less than $66.7 \%$ of the set constant voltage charge voltage. In the constant-voltage charging stage, the charging current is gradually reduced, the charging process continues until the charging current is reduced to zero, so that even if the solar cell output a very small power, but also the battery charge; because the battery terminal BAT pin voltage The same, so the battery will not be overcharged. When the input power is lost or the input voltage is lower than the battery voltage, the CN3722 automatically enters the low-power sleep mode. Other features include input low voltage latch, battery temperature monitoring, battery side overvoltage protection and charge status indication.
CN3722 uses constant pressure to track the maximum power point of solar cells. In the voltammetric characteristic curve of the solar cell, when the ambient temperature is constant, the output voltage corresponding to the output of the maximum power is basically the same at different sunshine intensities, that is, as long as the output voltage of the solar cell is kept constant, It is possible to ensure that the solar cell can output the maximum power when the light intensity is different at this temperature. When the ambient temperature changes, the voltage corresponding to the maximum power point of the solar cell varies with the temperature in accordance with the temperature coefficient of $-0.4 \% /{ }^{\circ} \mathrm{C}$, Formula to calculate the maximum power point. This maximum power point tracking method is very suitable for the four major temperature difference is relatively large or relatively large temperature difference between the situation, and our blinds are very consistent with the situation.

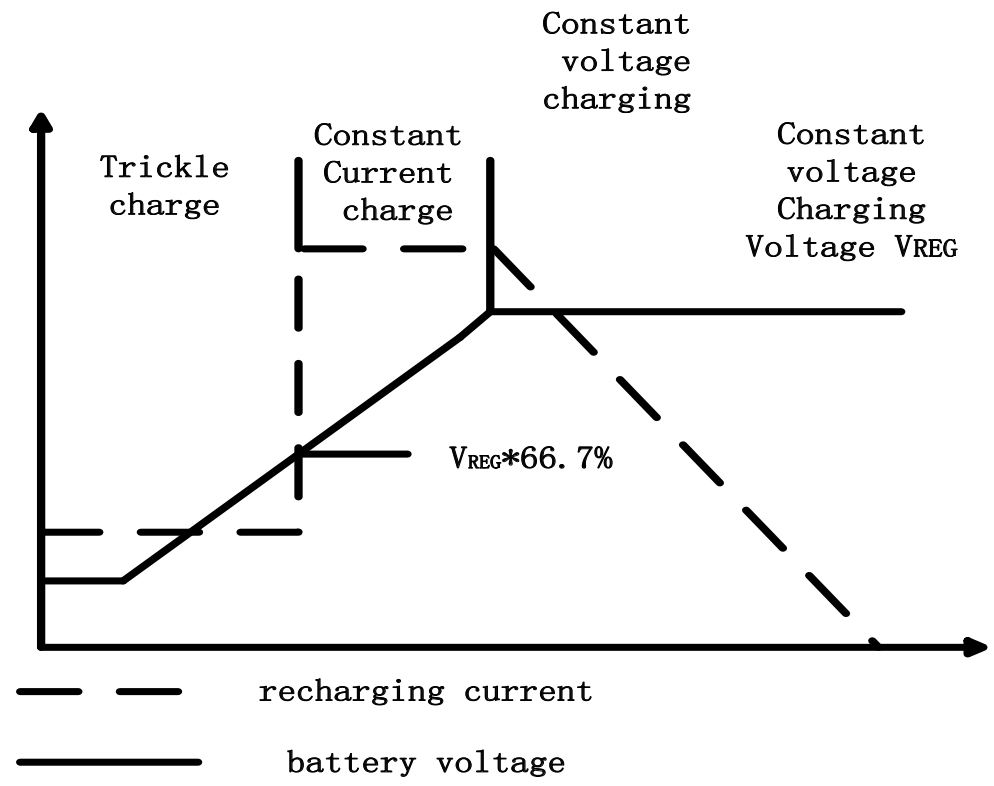

Figure 6 Schematic diagram of the charging process

\section{Software implementation}

The entire system to TMS320F28035 microcontroller as the control core, multi-sensor information fusion, the composition of the detection unit; click on the module composed of the implementation unit, according to the acquisition of voice and light information, effective, complete and reliable completion of the blinds open and automatic chasing light and other actions. The use of single-chip $\mathrm{C}$ voice programming and ccs compiler software, modular design ideas, is conducive to the commissioning procedures and follow-up maintenance upgrades. Design ideas as shown in the Figure 7 System program flow chart. Through the voice module to receive voice signals and 2.4-inch LCD serial port touch screen display the current working state. When a specific voice signal is received, the louver will perform the corresponding action until the next voice command is monitored. 


\section{System debugging}

\subsection{Speech recognition static test}

LD3320 speech recognition chip in the following two cases to give recognition results

(1) external into the scheduled time of the voice data, the chip after the analysis of these voice data analysis gives the results.

(2) external voice data stream, the voice recognition chip through the endpoint detection technology to detect the user to stop talking, and the user from the beginning to speak to stop the voice of the time period of the voice data analysis analysis gives the identification results.

The system designed in this paper is mainly used in voice control shutters, in the practical application of the main focus on voice recognition rate and real-time, so we use the second identification method. In order to better detect the recognition effect, we selected a number of different sound of the staff in the experimental environment several times to test. The results show that the average speech recognition rate is as high as $85 \%$ in the experimental environment.

\subsection{Test of the operation of the blinds in different power supply modes}

This experiment tested the effect of two different power supplies on the working condition of the blinds. Experimental results show that, regardless of the type of power supply, the blinds can work properly and complete the instructions

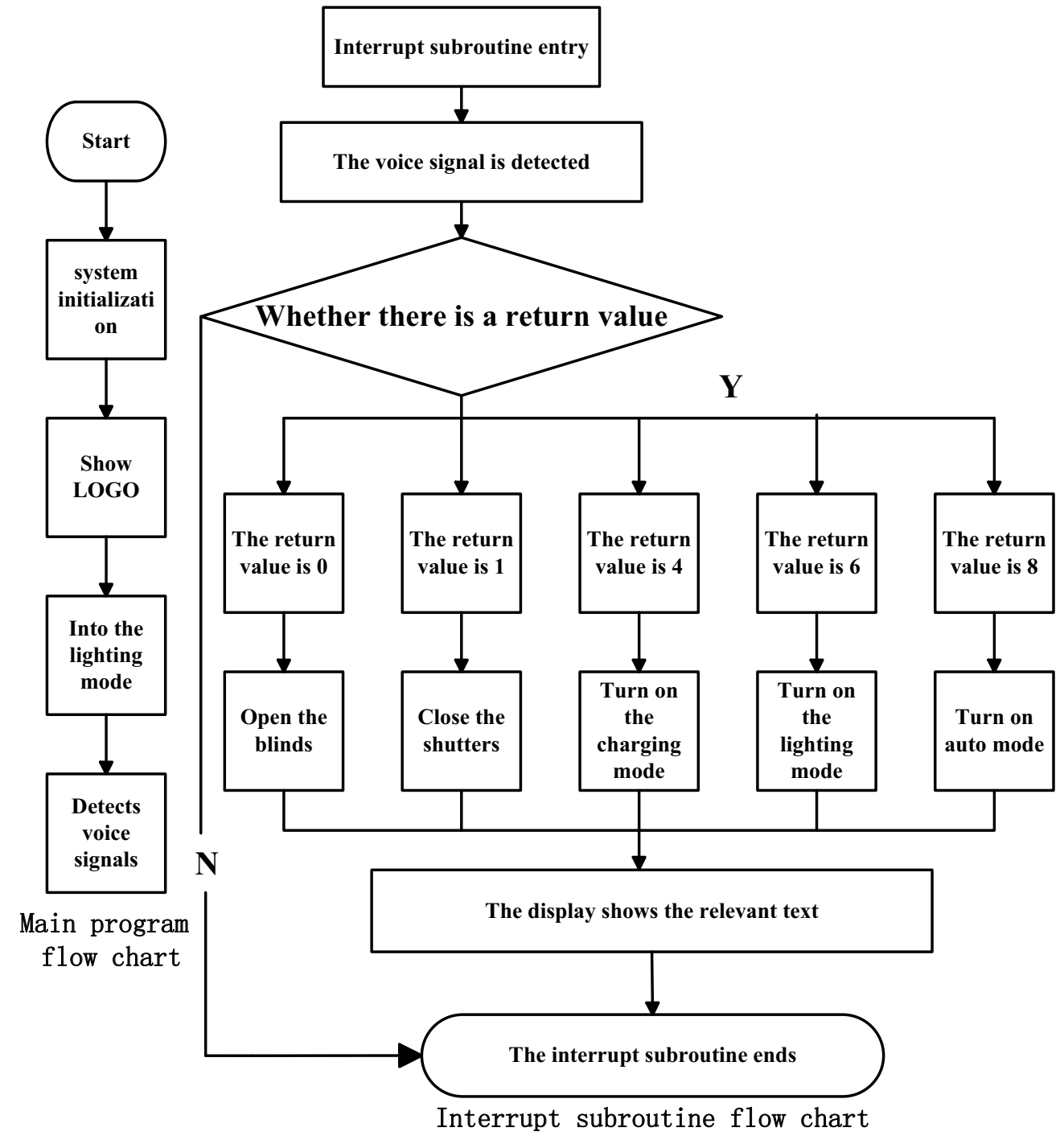

Figure 7 System program flow chart 


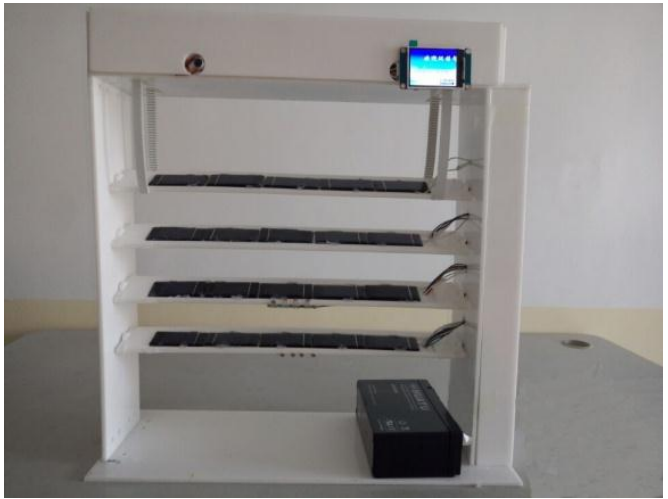

Figure 8 system test chart

\section{Conclusion}

Natural voice recognition, voice control and the rational and efficient use of natural resources is the focus of current research. This paper designs the DSP-based intelligent shutters voice control system to TMS320F28035 microcontroller as the control core, combined with the use of LD3320 chip and the use of CN3722 chip, completed the voice control and solar charging module design. Compared with the traditional blinds, we designed the intelligent blinds more in line with the current era of home intelligent and energy saving and environmental protection requirements, to facilitate people's lives. At present, there are still some shortcomings in the works, such as voice module recognition rate is very susceptible to external interference, but also need to further improve and deal with.

\section{references}

[1] Chen Xiaoyan, Pang Tao, Lian Ruoxin. Based on MCU multi-machine communication intelligent curtain design [J]. Measurement and Control Technology, 2012,31 (6): 76-78.

[2] Zhang Kairi, Deng Yanmei, Gao Shi. Photovoltaic power smart blinds system [Papers]. "Science and Technology Information", 2014, 21:34-37;

[3] Zheng Zhenjie, Jiang Yanxuan, Yu Dezhi. Singlechip microcomputer combined with ULN2003 driven stepper motor. "Motor Technology", 2011,31:27-32.

[4] Chen Boshi. Electric drive automatic control system. Third edition. Beijing: Mechanical Industry Press, 2011

[5] $\mathrm{Hu}$ Shousong. The principle of automatic control. Fifth edition. Beijing: Science Press, 2007

[6] Xu Dehong. Modeling and control of power electronic systems. First edition. Beijing: China Machine Press, 2006

Foundation item: the project by science and Technology Department of Hebei province (1627460316214408); Chinese Coal Industry Association (MTKJ2016-35, MTKJ2016-329); fundamental research funds for the central universities such as funding (3142013101, 3142016022) 